\title{
Sensitivity and speed of colour Doppler flow mapping compared with continuous wave Doppler for the detection of ventricular septal defects
}

\author{
David T Linker, Ole Rossvoll, James V Chapman, Bjørn A J Angelsen
}

\begin{abstract}
Twenty nine patients (aged from three months to 37 years) with confirmed or suspected ventricular septal defects were studied separately by three examiners who used colour flow mapping and imaging, or continuous wave Doppler and imaging, or a combined reference examination. Colour flow mapping identified 19 of the 25 patients with a ventricular septal defect, continuous wave Doppler echocardiography identified 18, and the combined reference examination identified 24. Two of four patients without ventricular septal defect had a false positive result with colour flow mapping and none had a false positive result with continuous wave Doppler examination. During the reference examination continuous wave Doppler identified 24 patients with ventricular septal defects and colour flow mapping identified 23. In two patients a second ventricular septal defect was found by colour flow mapping, and confirmed by continuous wave Doppler. There was no significant difference in time to diagnosis between the two techniques.

Colour flow mapping aids identification of multiple ventricular septal defects but is not faster and has lower specificity than continuous wave Doppler. A combination of the two techniques gave the highest sensitivity and specificity.
\end{abstract}

Department of Biomedical Engineering and Division of Cardiology, University of Trondheim, Trondheim, Norway D T Linker O Rossvoll

J C Chapman

B A J Angelsen

Correspondence to Dr David T Linker, Department of University of Trondheim, 7006 Trondheim, Norway.

Accepted for publication 20 November 1990
Doppler examine only a given direction or point, respectively. For this reason, it has been suggested that colour flow mapping would be helpful in screening for lesions such as ventricular septal defects.
The current study was designed to compare the speed and accuracy of colour flow mapping and continuous wave Doppler for the diagnosis of ventricular septal defects when both are used in conjunction with cross sectional echocardiography.

\section{Patients and methods}

PATIENT SELECTION

We reviewed patient records or referrals for the diagnosis of suspected or actual ventricular septal defect and selected 29 outpatients, aged three months to 37 years (mean (SD) 6.2 $(8 \cdot 3)$ years). The distribution of diagnoses was 11 infracristal/perimembranous (one with moderate pulmonary stenosis), one supracristal, 10 muscular/trabecular (one associated with Ebstein's anomaly), two tetralogy of Fallot, one pulmonary atresia with ventricular septal defect, and four closed spontaneously or at operation. Patients with atrioventricular canal were excluded from the study. Only three patients had been catheterised.

\section{EXAMINERS}

We chose two test examiners who had more experience in the diagnosis of adult heart disease than with infants and children. The first test examiner was a technician with a total of 11 years' experience of echocardiography, ten years with Doppler echocardiography, and two with colour flow mapping, who used only cross sectional imaging and colour flow mapping to make a diagnosis. The second test examiner was a cardiology fellow with one year's experience of Doppler echocardiography, who used only cross sectional imaging and continuous wave Doppler to make a diagnosis. The third, or reference examiner, was a paediatric cardiologist with five years' experience in echocardiography/Doppler, who used the history, chart, physical examination, and cross sectional echocardiography, continuous wave Doppler, and colour flow mapping to reach the reference diagnosis. This examiner also used all ultrasound methods together and compared the optimal results with colour flow mapping and continuous wave Doppler.

\section{EQUIPMENT}

All examinations were performed with a mechanical, annular array, colour flow mapping system (CFM-700, Vingmed Sound, A/S). 
This system allows all the Doppler methods to be achieved with the same imaging transducer (3.5 $\mathrm{MHz}$ ) and it also allows simultaneous imaging and continuous wave Doppler examination.

\section{EXAMINATION PROTOCOL}

Unsedated patients were examined independently by the test examiners. The examination was stopped when an experimental examiner and the reference examiner agreed that the diagnostic criteria were met, or after 15 minutes. The time and diagnosis were recorded for each examination. If there was no conclusion, an examination time of 15 minutes was recorded. Unlike the reference examiner, the experimental examiners did not have access to any information about the patient or the results of other examinations, other than that the patient had a suspected ventricular septal defect.

After both of the experimental examiners completed their examinations, the reference examiner used all available data, and supplementary echocardiographic or Doppler examinations when necessary, to determine the final diagnosis. During this final examination, he attempted to show the lesion by both methods, if necessary using the other as a guide to determine the absolute sensitivity.

The diagnostic criteria for a ventricular septal defect were as follows: for flow mapping the presence of flow that clearly passed through the ventricular septum, systolic disturbed flow ("mosaic pattern") on the right side of the septum, or a clear defect on the imaging examination were each considered sufficient; for the continuous wave Doppler examinationsystolic flow directed towards the transducer with a velocity $>3 \mathrm{~m} / \mathrm{s}$, or a clear defect on cross sectional echocardiography were each considered sufficient. These criteria for continuous wave Doppler examination would miss patients with high right sided pressures and a ventricular septal defect too small to be seen on cross sectional imaging; however, none of the patients in the study population had this combination of features.

The classification of the patients was based on a total evaluation by the reference examiner. But the results of the reference examination were based on a strict interpretation of the reference examiner's results using the criteria of the study, which meant that both false positive and negative results were possible.

There were no disagreements over the diagnoses. In two patients the time to diagnosis was extended for the flow mapping examination

Number of positive and negative diagnostic examinations for each combination of actual diagnosis (left column) and type of examination

\begin{tabular}{|c|c|c|c|c|c|c|c|c|}
\hline \multirow[b]{3}{*}{ Diagnosis } & \multirow{2}{*}{\multicolumn{2}{|c|}{$\begin{array}{l}\text { Colour flow } \\
\text { mapping }\end{array}$}} & \multirow{2}{*}{\multicolumn{2}{|c|}{$\begin{array}{l}\text { Continuous } \\
\text { wave }\end{array}$}} & \multicolumn{4}{|c|}{ Reference examiner } \\
\hline & & & & & \multicolumn{2}{|c|}{$\begin{array}{l}\text { Colour flow } \\
\text { mapping }\end{array}$} & \multicolumn{2}{|c|}{$\begin{array}{l}\text { Continuous } \\
\text { wave }\end{array}$} \\
\hline & Pos & Neg & Pos & $\mathrm{Neg}$ & Pos & $\mathrm{Neg}$ & Pos & $\mathrm{Neg}$ \\
\hline $\begin{array}{l}\text { VSD } \\
\text { No VSD }\end{array}$ & $\begin{array}{r}19 \\
2\end{array}$ & $\begin{array}{l}6 \\
2\end{array}$ & $\begin{array}{r}18 \\
0\end{array}$ & $\begin{array}{l}7 \\
4\end{array}$ & $\begin{array}{r}23 \\
0\end{array}$ & $\begin{array}{l}2 \\
4\end{array}$ & $\begin{array}{r}24 \\
0\end{array}$ & $\begin{array}{l}1 \\
4\end{array}$ \\
\hline
\end{tabular}

VSD, ventricular septal defect. because a test examiner and the reference examiner did not agree that the diagnostic criteria had been met. The examiners generally used the parasternal and subcostal approach but individual examiners were free to use whatever view they wished.

\section{STATISTICAL ANALYSIS}

The times that it took to reach a diagnosis for each patient with continuous wave Doppler and colour flow mapping were compared by Student's paired $t$ test.

\section{Results}

The table shows the diagnostic results. In 25 patients a ventricular septal defect was the final diagnosis. Of these, 19 were diagnosed by colour flow mapping and 18 by continuous wave Doppler. Of the patients with a false negative diagnosis on both examinations, one had pulmonary atresia and low velocity through a moderate sized defect, one had a supracristal defect, and four had muscular defects. The one patient in whom a ventricular septal defect was detected by flow mapping but not by continuous wave had a muscular defect.

All four patients without ventricular septal defects were correctly classified by continuous wave Doppler examination, but in two colour flow mapping incorrectly identified ventricular septal defects. Both of the false positive diagnoses arose because disturbed flow on the right side of the septum simulated a small muscular ventricular septal defect.

All except one patient with a ventricular septal defect were identified when the reference examiner used continuous wave Doppler echocardiography. The false negative result arose in a patient with a supracristal defect with flow directed towards the pulmonary valve that did not meet any of the diagnostic criteria. Twenty three defects were identified by colour flow mapping. The additional false negative was a small muscular defect. The small number of false positives and false negatives in the test and reference examinations precluded statistical testing for differences.

The time to diagnosis was $4 \cdot 8(5 \cdot 9)$ minutes for the colour flow mapping examination and $5.8(5.9)$ minutes for the continuous wave Doppler examination. This difference was not statistically significant. The mean difference in time to diagnosis between the two techniques was $0.9(3.4)$ minutes, and it was not significantly different from zero $(p>0.2)$. In only three patients was a conclusion reached after more than five minutes (maximum 11 minutes) of flow mapping, and similarly, only three patients needed more than five minutes of examination by continuous wave Doppler echocardiography (maximum eight minutes).

We found a second, unsuspected septal defect by colour flow mapping in two of the patients with a ventricular septal defect; both were confirmed by continuous wave Doppler examination. In one the second defect was from the left ventricle to right atrium and seemed to be more important than the original defect; the other was a small muscular defect. 


\section{Discussion}

The addition of pulsed Doppler to cross sectional imaging has improved the diagnostic sensitivity of echocardiography for ventricular septal defects. ${ }^{1-3}$ Continuous wave Doppler has also been used to diagnose ventricular septal defects, and it also gives information about physiological variables. ${ }^{4-7}$

Colour Doppler flow mapping has been used to identify ventricular septal defects, ${ }^{89}$ and it seems to be especially good at identifying multiple ventricular septal defects. ${ }^{10}$ It can clearly show the pattern of flow both through the septum and into the other heart chamber. The relative sensitivity of the various techniques is unknown, however. In the hands of experienced examiners both pulsed Doppler and colour flow mapping were highly sensitive and specific for the detection of ventricular septal defects in patients selected for catheterisation. ${ }^{10}$ This population tended to have larger defects, and it seems reasonable to assume that errors would be more likely with the smaller lesions that are seen in a less selected patient group.

We found that the identification of ventricular septal defects by an experienced examiner with continuous wave Doppler was easy in almost all patients, whereas pulsed Doppler tends to be more difficult, leading to more potential false negatives. ${ }^{11}$ One reason for this is that all depths in the direction of the beam are examined simultaneously by continuous wave Doppler; with pulsed Doppler the sample volume must be moved to each depth. Any improvement in speed and accuracy with colour flow mapping over the continuous wave examination is more likely in observers who were less experienced in congenital heart disease and who would be less certain of the continuous wave examination. We found that the continuous wave Doppler examination performed by such an examiner was as sensitive, fast, and may have been less liable to give false positive results than the flow mapping examination. On the other hand, the colour flow mapping examination identified previously unknown multiple ventricular septal defects. The best approach to achieve the highest sensitivity and specificity would be to use the two techniques to supplement one another.

The reference examiner had one false negative continuous wave Doppler examination. This was in a patient with a supracristal ventricular septal defect that caused turbulent, high velocity flow through the pulmonary valve and simulated pulmonary stenosis; it did not meet any of the diagnostic criteria of the study. Another false negative on cross sectional Doppler examination was caused by a small apical defect. The cause of the false positives on cross sectional Doppler examination is unclear, but one potential cause was flow disturbance around right ventricular septal trabeculae.

The greater sensitivity of the continuous wave Doppler examination in experienced hands for finding ventricular septal defects may be because it has a better time resolution than colour flow mapping.

For this study we used a single model of ultrasound machine and transducer with only one colour flow map. Because the use of different colour flow maps will change the operator's perception of normal and disturbed flow it can influence sensitivity. Clearly, the characteristics of the transducer and instrument also affect the absolute sensitivity of the techniques as well as their relative sensitivity. This means that our results cannot be extrapolated to all combinations of instrument and transducer, though in our opinion the intrinsic characteristics of colour Doppler flow mapping and continuous wave Doppler account for their relative strengths and weaknesses.

1 Magherini A, Simonetti L, Tomassini CR, Moggi C, Ragazzini F, Bartolozzi G. Cross-sectional echocardiography with pulsed and continuous wave Doppler in the management of ventricular septal defects. Int J Cardiol 1987;15:317-28.

2 Smith G, Endresen K, Sivertssen E, Semb G. Ventricular septal rupture diagnosed by simultaneous cross-sectional echocardiography and Doppler ultrasound. Eur Heart $J$ 1985;6:631-6.

3 Stevenson JG, Kawabori I, Stamm SJ, et al. Pulsed Doppler echocardiographic evaluation of ventricular septal defect patches. Circulation 1984;70(suppl 1):38-46.

4 patches. Circulation 1984;70(suppl 1):38-46. of ventricular septal defect by Doppler ultrasound. Acta Med Scand 1981;suppl 645:47-56.

5 Marx GR, Allen HD, Goldberg SJ. Doppler echocardiographic estimation of systolic pulmonary artery pressure in pediatric patients with interventricular communications. J Am Coll Cardiol 1985;6:1132-7.

6 Murphy DJ, Ludomirsky A, Huhta JC. Continuous-wave Doppler in children with ventricular septal defect: noninvasive estimation of interventricular pressure gradient. Am J Cardiol 1986;57:428-32.

7 Stevenson JG. Doppler evaluation of atrial septal defect, ventricular septal defect, and complex malformations. Acta Paediatr Scand 1986;supp1 329:21-43.

8 Ortiz E, Robinson PJ, Deanfield JE, Franklin R, Macartney FJ, Wyse RKH. Localisation of ventricular septal defects by simultaneous display of superimposed colour Doppler by simultaneous display of superimposed colour Doppler
and cross sectional echocardiographic images. Br Heart J and cross section

9 Ritter SB. Two-dimensional Doppler color flow mapping in congenital heart disease. Clin Cardiol 1986;9:591-6.

10 Ludomirsky A, Huhta JC, Vick GW III, Murphy DJ, Danford DA, Morrow WR. Color Doppler detection of multiple ventricular septal defects. Circulation 1986; 74:1317-22.

11 Kapusta L, Hopman JCW, Daniels O. The usefulness of cross-sectional Doppler flow imaging in the detection of small ventricular septal defects with left-to-right shunt. Eur Heart J 1987;8:1002-6. 